\title{
HYPERSURFACES OF PRESCRIBED MEAN CURVATURE IN LORENTZIAN MANIFOLDS
}

\author{
CLAUS GERHARDT
}

\begin{abstract}
We give a new existence proof for closed hypersurfaces of prescribed mean curvature in Lorentzian manifolds.
\end{abstract}

\section{Contents}

0. Introduction 1

1. Notations and definitions 2

2. The evolution problem 5

3. Lower order estimates 8

4. $C^{2}$-estimates 13

5. Convergence to a stationary solution 14

References 15

\section{INTRODUCTION}

Hypersurfaces of prescribed mean curvature especially those with constant mean curvature play an important role in general relativity. In 3] the existence of closed hypersurfaces of prescribed mean curvature in a globally hyperbolic Lorentz manifold with a compact Cauchy hypersurface was proved provided there were barriers. The proof consisted of two parts, the a priori estimates for the gradient and the application of a fixed point theorem. That latter part of the proof was rather complicated, and certainly nobody would have qualified it as elegant.

Ecker and Huisken, therefore, gave another existence proof using an evolutionary approach, but they had to assume that the time-like convergence condition is satisfied, and, even more important, that the prescribed mean curvature satisfies a structural monotonicity condition, cf. 2. These are serious restrictions which had to be assumed because the

Date: April 12, 1999.

Key words and phrases. Prescribed mean curvature, Lorentz manifold. 
authors relied on the gradient estimate of Bartnik [1, who had proved another a priori estimate in the elliptic case.

We shall show in the following that the evolutionary method can be used in the existence proof without any unnecessary restrictions on the curvature of the ambient space or the right-hand side. The only difference in the assumptions - relative to our former paper - is that the right-hand side is now supposed to be of class $C^{1}$, while bounded is actually sufficient. But this drawback can easily be overcome by approximation.

This paper is organized as follows: In Section 1 we introduce the notations and definitions we rely on.

In Section 2 we look at the curvature flow associated with our problem, and the corresponding evolution equations for the basic geometric quantities of the flow hypersurfaces.

In Section 3 lower order estimates for the evolution problem are proved, while a priori estimates in the $C^{2}$-norm are derived in Section 4

Finally, in Section 5. we demonstrate that the evolutionary solution converges to a stationary solution.

\section{Notations And Definitions}

The main objective of this section is to state the equations of Gauß, Codazzi, and Weingarten for hypersurfaces $M$ in a $(\mathrm{n}+1)$-dimensional Lorentzian space $N$. Geometric quantities in $N$ will be denoted by $\left(\bar{g}_{\alpha \beta}\right),\left(\bar{R}_{\alpha \beta \gamma \delta}\right)$, etc., and those in $M$ by $\left(g_{i j}\right),\left(R_{i j k l}\right)$, etc. Greek indices range from 0 to $n$ and Latin from 1 to $n$; the summation convention is always used. Generic coordinate systems in $N$ resp. $M$ will be denoted by $\left(x^{\alpha}\right)$ resp. $\left(\xi^{i}\right)$. Covariant differentiation will simply be indicated by indices, only in case of possible ambiguity they will be preceded by a semicolon, i.e. for a function $u$ in $N,\left(u_{\alpha}\right)$ will be the gradient and $\left(u_{\alpha \beta}\right)$ the Hessian, but e.g., the covariant derivative of the curvature tensor will be abbreviated by $\bar{R}_{\alpha \beta \gamma \delta ; \epsilon}$. We also point out that

$$
\bar{R}_{\alpha \beta \gamma \delta ; i}=\bar{R}_{\alpha \beta \gamma \delta ; \epsilon} x_{i}^{\epsilon}
$$

with obvious generalizations to other quantities.

Let $M$ be a space-like hypersurface, i.e. the induced metric is Riemannian, with a differentiable normal $\nu$ that is time-like.

In local coordinates, $\left(x^{\alpha}\right)$ and $\left(\xi^{i}\right)$, the geometric quantities of the space-like hypersurface $M$ are connected through the following equations

$$
x_{i j}^{\alpha}=h_{i j} \nu^{\alpha}
$$


the so-called Gauß formula. Here, and also in the sequel, a covariant derivative is always a full tensor, i.e.

$$
x_{i j}^{\alpha}=x_{, i j}^{\alpha}-\Gamma_{i j}^{k} x_{k}^{\alpha}+\bar{\Gamma}_{\beta \gamma}^{\alpha} x_{i}^{\beta} x_{j}^{\gamma} .
$$

The comma indicates ordinary partial derivatives.

In this implicit definition the second fundamental form $\left(h_{i j}\right)$ is taken with respect to $\nu$.

The second equation is the Weingarten equation

$$
\nu_{i}^{\alpha}=h_{i}^{k} x_{k}^{\alpha},
$$

where we remember that $\nu_{i}^{\alpha}$ is a full tensor.

Finally, we have the Codazzi equation

$$
h_{i j ; k}-h_{i k ; j}=\bar{R}_{\alpha \beta \gamma \delta} \nu^{\alpha} x_{i}^{\beta} x_{j}^{\gamma} x_{k}^{\delta}
$$

and the Gauß equation

$$
R_{i j k l}=-\left\{h_{i k} h_{j l}-h_{i l} h_{j k}\right\}+\bar{R}_{\alpha \beta \gamma \delta} x_{i}^{\alpha} x_{j}^{\beta} x_{k}^{\gamma} x_{l}^{\delta} .
$$

Now, let us assume that $N$ is a globally hyperbolic Lorentzian manifold with a compact Cauchy surface. $N$ is then a topological product $\mathbb{R} \times \mathcal{S}_{0}$, where $\mathcal{S}_{0}$ is a compact Riemannian manifold, and there exists a Gaussian coordinate system $\left(x^{\alpha}\right)$, such that the metric in $N$ has the form

$$
d \bar{s}_{N}^{2}=e^{2 \psi}\left\{-d x^{0^{2}}+\sigma_{i j}\left(x^{0}, x\right) d x^{i} d x^{j}\right\},
$$

where $\sigma_{i j}$ is a Riemannian metric, $\psi$ a function on $N$, and $x$ an abbreviation for the space-like components $\left(x^{i}\right)$, see [8], 10, p. 212], 9, p. 252], and [3. Section 6]. We also assume that the coordinate system is future oriented, i.e. the time coordinate $x^{0}$ increases on future directed curves. Hence, the contravariant time-like $\operatorname{vector}\left(\xi^{\alpha}\right)=(1,0, \ldots, 0)$ is future directed as is its covariant version $\left(\xi_{\alpha}\right)=e^{2 \psi}(-1,0, \ldots, 0)$.

Let $M=\operatorname{graph} u_{\left.\right|_{\mathcal{S}_{0}}}$ be a space-like hypersurface

$$
M=\left\{\left(x^{0}, x\right): x^{0}=u(x), x \in \mathcal{S}_{0}\right\},
$$

then the induced metric has the form

$$
g_{i j}=e^{2 \psi}\left\{-u_{i} u_{j}+\sigma_{i j}\right\}
$$

where $\sigma_{i j}$ is evaluated at $(u, x)$, and its inverse $\left(g^{i j}\right)=\left(g_{i j}\right)^{-1}$ can be expressed as

$$
g^{i j}=e^{-2 \psi}\left\{\sigma^{i j}+\frac{u^{i}}{v} \frac{u^{j}}{v}\right\}
$$


where $\left(\sigma^{i j}\right)=\left(\sigma_{i j}\right)^{-1}$ and

$$
\begin{aligned}
u^{i} & =\sigma^{i j} u_{j} \\
v^{2} & =1-\sigma^{i j} u_{i} u_{j} \equiv 1-|D u|^{2} .
\end{aligned}
$$

Hence, graph $u$ is space-like if and only if $|D u|<1$.

The covariant form of a normal vector of a graph looks like

$$
\left(\nu_{\alpha}\right)= \pm v^{-1} e^{\psi}\left(1,-u_{i}\right) .
$$

and the contravariant version is

$$
\left(\nu^{\alpha}\right)=\mp v^{-1} e^{-\psi}\left(1, u^{i}\right) .
$$

Thus, we have

1.1. Remark. Let $M$ be space-like graph in a future oriented coordinate system. Then, the contravariant future directed normal vector has the form

$$
\left(\nu^{\alpha}\right)=v^{-1} e^{-\psi}\left(1, u^{i}\right)
$$

and the past directed

$$
\left(\nu^{\alpha}\right)=-v^{-1} e^{-\psi}\left(1, u^{i}\right)
$$

In the Gauß formula (1.2) we are free to choose the future or past directed normal, but we stipulate that we always use the past directed normal for reasons that we have explained in 6 .

Look at the component $\alpha=0$ in (1.2) and obtain in view of (1.15)

$$
e^{-\psi} v^{-1} h_{i j}=-u_{i j}-\bar{\Gamma}_{00}^{0} u_{i} u_{j}-\bar{\Gamma}_{0 j}^{0} u_{i}-\bar{\Gamma}_{0 i}^{0} u_{j}-\bar{\Gamma}_{i j}^{0} .
$$

Here, the covariant derivatives a taken with respect to the induced metric of $M$, and

$$
-\bar{\Gamma}_{i j}^{0}=e^{-\psi} \bar{h}_{i j}
$$

where $\left(\bar{h}_{i j}\right)$ is the second fundamental form of the hypersurfaces $\left\{x^{0}=\right.$ const\}.

An easy calculation shows

$$
\bar{h}_{i j} e^{-\psi}=-\frac{1}{2} \dot{\sigma}_{i j}-\dot{\psi} \sigma_{i j},
$$

where the dot indicates differentiation with respect to $x^{0}$.

Next, let us analyze under which condition a space-like hypersurface $M$ can be written as a graph over the Cauchy hypersurface $\mathcal{S}_{0}$.

We first need 
1.2. Definition. Let $M$ be a closed, space-like hypersurface in $N$. Then,

(i) $M$ is said to be achronal, if no two points in $M$ can be connected by a future directed time-like curve.

(ii) $M$ is said to separate $N$, if $N \backslash M$ is disconnected.

In [6. Proposition 2.5] we proved

1.3. Proposition. Let $N$ be connected and globally hyperbolic, $\mathcal{S}_{0} \subset N$ a compact Cauchy hypersurface, and $M \subset N$ a compact, connected space-like hypersurface of class $C^{m}, m \geq 1$. Then, $M=\operatorname{graph} u_{\left.\right|_{\mathcal{S}_{0}}}$ with $u \in C^{m}\left(\mathcal{S}_{0}\right)$ iff $M$ is achronal.

Sometimes, we need a Riemannian reference metric, e.g. if we want to estimate tensors. Since the Lorentzian metric can be expressed as

$$
\bar{g}_{\alpha \beta} d x^{\alpha} d x^{\beta}=e^{2 \psi}\left\{-d x^{0^{2}}+\sigma_{i j} d x^{i} d x^{j}\right\},
$$

we define a Riemannian reference metric $\left(\tilde{g}_{\alpha \beta}\right)$ by

$$
\tilde{g}_{\alpha \beta} d x^{\alpha} d x^{\beta}=e^{2 \psi}\left\{d x^{0^{2}}+\sigma_{i j} d x^{i} d x^{j}\right\}
$$

and we abbreviate the corresponding norm of a vectorfield $\eta$ by

$$
\|\eta \mid\|=\left(\tilde{g}_{\alpha \beta} \eta^{\alpha} \eta^{\beta}\right)^{1 / 2},
$$

with similar notations for higher order tensors.

\section{The EVOlution PROBlem}

Let $N$ be a globally hyperbolic Lorentzian manifold with a compact Cauchy hypersurface $\mathcal{S}_{0}$. Consider the problem of finding a closed hypersurface of prescribed mean curvature $H$ in $N$, or more precisely, let $\Omega$ be a connected open subset of $N, f \in C^{0, \alpha}(\bar{\Omega})$, then we look for a hypersurface $M \subset \Omega$ such that

$$
H_{\left.\right|_{M}}=f(x) \quad \forall x \in M,
$$

where $H_{\left.\right|_{M}}$ means that $H$ is evaluated at the vector $\left(\kappa_{i}(x)\right)$ the components of which are the principal curvatures of $M$.

We assume that $\partial \Omega$ consists of two achronal, compact, connected, space-like hypersurfaces $M_{1}$ and $M_{2}$, where $M_{1}$ is supposed to lie in the past of $M_{2}$. The $M_{i}$ should act as barriers for $(H, f)$.

2.1. Definition. $M_{2}$ is an upper barrier for $(H, f)$, if $M_{2}$ is of class $C^{2, \alpha}$ and

$$
H_{\left.\right|_{M_{2}}} \geq f
$$


and $M_{1}$ ia a lower barrier for $(H, f)$, if $M_{1}$ is of class $C^{2, \alpha}$ satisfying

$$
H_{\left.\right|_{M_{1}}} \leq f
$$

In [3, Section 6] we proved the following theorem

2.2. Theorem. Let $M_{1}$ be a lower and $M_{2}$ be an upper barrier for $(H, f), f \in C^{0, \alpha}(\bar{\Omega})$. Then, the problem

$$
H_{\left.\right|_{M}}=f
$$

has a solution $M \subset \bar{\Omega}$ of class $C^{2, \alpha}$ that can be written as a graph over the Cauchy hypersurface $\mathcal{S}_{0}$.

The crucial point in the proof is an a priori estimate in the $C^{1}$-norm and for this estimate only the boundedness of $f$ is needed, i.e. even for merely bounded $f H^{2, p}$ solutions exist.

We want to give a new proof of Theorem 2.2 that is based on the evolution method, and to make this method work, we have to assume temporarily slightly higher degrees of regularity for the barriers and right-hand side, i.e. we assume the barriers to be of class $C^{4, \alpha}$ and $f$ to be of class $C^{2, \alpha}$. We can achieve these assumptions by approximation without sacrificing the barrier conditions, cf. [5. p. 179].

To solve (2.4) we look at the evolution problem

$$
\begin{aligned}
\dot{x} & =(H-f) \nu, \\
x(0) & =x_{0},
\end{aligned}
$$

where $x_{0}$ is an embedding of an initial hypersurface $M_{0}$, for which we choose $M_{0}=M_{2}, H$ is the mean curvature of the flow hypersurfaces $M(t)$ with respect to the past directed normal $\nu$, and $x(t)$ is an embedding of $M(t)$.

In 6] we have considered problems of the form (2.5) for general curvature operators in a pseudo-riemannian setting, so that the present situation can be retrieved as a special case of the general results in 6. Section 3].

The evolution exists on a maximal time interval $\left[0, T^{*}\right), 0<T^{*} \leq \infty$, cf. [4, Section 2], where we apologize for the ambiguity of also calling the evolution parameter time.

Next, we want to show how the metric, the second fundamental form, and the normal vector of the hypersurfaces $M(t)$ evolve. All time derivatives are total derivatives. We refer to [6] for more general results and to [4. Section 3], where proofs are given in a Riemannian setting, but these proofs are also valid in a Lorentzian environment. 
2.3. Lemma. The metric, the normal vector, and the second fundamental form of $M(t)$ satisfy the evolution equations

$$
\dot{\nu}=\nabla_{M}(H-f)=g^{i j}(H-f)_{i} x_{j},
$$

and

$$
\begin{gathered}
\dot{h}_{i}^{j}=(H-f)_{i}^{j}-(H-f) h_{i}^{k} h_{k}^{j}-(H-f) \bar{R}_{\alpha \beta \gamma \delta} \nu^{\alpha} x_{i}^{\beta} \nu^{\gamma} x_{k}^{\delta} g^{k j} \\
\dot{h}_{i j}=(H-f)_{i j}+(H-f) h_{i}^{k} h_{k j}-(H-f) \bar{R}_{\alpha \beta \gamma \delta} \nu^{\alpha} x_{i}^{\beta} \nu^{\gamma} x_{j}^{\delta} .
\end{gathered}
$$

2.4. Lemma (Evolution of $(H-f))$. The term $(H-f)$ evolves according to the equation

$$
\begin{aligned}
(H-f)^{\prime}-\Delta(H-f)= & -\|A\|^{2}(H-f)-f_{\alpha} \nu^{\alpha}(H-f) \\
& -\bar{R}_{\alpha \beta} \nu^{\alpha} \nu^{\beta}(H-f),
\end{aligned}
$$

where

$$
(H-f)^{\prime}=\frac{d}{d t}(H-f)
$$

and

$$
\|A\|^{2}=h_{i j} h^{i j}
$$

From (2.8) we deduce with the help of the Ricci identities a parabolic equation for the second fundamental form

2.5. Lemma. The mixed tensor $h_{i}^{j}$ satisfies the parabolic equation

$$
\begin{aligned}
\dot{h}_{i}^{j}-\Delta h_{i}^{j}= & -\|A\|^{2} h_{i}^{j}+f h_{i}^{k} h_{k}^{j}-f_{\alpha \beta} x_{i}^{\alpha} x_{k}^{\beta} g^{k j}-f_{\alpha} \nu^{\alpha} h_{i}^{j} \\
& +2 \bar{R}_{\alpha \beta \gamma \delta} x_{m}^{\alpha} x_{i}^{\beta} x_{k}^{\gamma} x_{r}^{\delta} h^{k m} g^{r j} \\
& -g^{k l} \bar{R}_{\alpha \beta \gamma \delta} x_{m}^{\alpha} x_{k}^{\beta} x_{r}^{\gamma} x_{l}^{\delta} h_{i}^{m} g^{r j}-g^{k l} \bar{R}_{\alpha \beta \gamma \delta} x_{m}^{\alpha} x_{k}^{\beta} x_{i}^{\gamma} x_{l}^{\delta} h^{m j} \\
& -\bar{R}_{\alpha \beta} \nu^{\alpha} \nu^{\beta} h_{i}^{j}+f \bar{R}_{\alpha \beta \gamma \delta} \nu^{\alpha} x_{i}^{\beta} \nu^{\gamma} x_{m}^{\delta} g^{m j} \\
& +g^{k l} \bar{R}_{\alpha \beta \gamma \delta ; \epsilon}\left\{\nu^{\alpha} x_{k}^{\beta} x_{l}^{\gamma} x_{i}^{\delta} x_{m}^{\epsilon} g^{m j}+\nu^{\alpha} x_{i}^{\beta} x_{k}^{\gamma} x_{m}^{\delta} x_{l}^{\epsilon} g^{m j}\right\} .
\end{aligned}
$$

2.6. Remark. In view of the maximum principle, we immediately deduce from (2.10) that the term $(H-f)$ has a sign during the evolution if it has one at the beginning. Thus, we have

$$
H \geq f \text {. }
$$




\section{LOWER ORDER ESTIMATES}

We recall our assumption that the ambient space is globally hyperbolic with a compact Cauchy hypersurface $\mathcal{S}_{0}$. The barriers $M_{i}$ are then graphs over $\mathcal{S}_{0}, M_{i}=$ graph $u_{i}$, because they are achronal, cf. Proposition 1.3, and we have

$$
u_{1} \leq u_{2},
$$

for $M_{1}$ should lie in the past of $M_{2}$, and the enclosed domain is supposed to be connected. Moreover, in view of the Harnack inequality, the strict inequality is valid in (3.1) unless the barriers coincide and are a solution to our problem.

Let us look at the evolution equation (2.5) with initial hypersurface $M_{0}$ equal to $M_{2}$. Then, because of the short-time existence, the evolution will exist on a maximal time interval $I=\left[0, T^{*}\right), T^{*} \leq \infty$, as long as the evolving hypersurfaces are space-like and smooth.

Furthermore, since the initial hypersurface is a graph over $\mathcal{S}_{0}$, we can write

$$
M(t)=\operatorname{graph} u(t)_{\left.\right|_{S_{0}}} \quad \forall t \in I,
$$

where $u$ is defined in the cylinder $Q_{T^{*}}=I \times \mathcal{S}_{0}$. We then deduce from (2.5), looking at the component $\alpha=0$, that $u$ satisfies a parabolic equation of the form

$$
\dot{u}=-e^{-\psi} v^{-1}(H-f),
$$

where we use the notations in Section 11, and where we emphasize that the time derivative is a total derivative, i.e.

$$
\dot{u}=\frac{\partial u}{\partial t}+u_{i} \dot{x}^{i}
$$

Since the past directed normal can be expressed as

$$
\left(\nu^{\alpha}\right)=-e^{-\psi} v^{-1}\left(1, u^{i}\right),
$$

we conclude from (2.5), (3.3), and (3.4)

$$
\frac{\partial u}{\partial t}=-e^{-\psi} v(H-f) \text {. }
$$

Thus, $\frac{\partial u}{\partial t}$ is non-positive in view of Remark 2.6 .

Next, let us state our first a priori estimate

3.1. Lemma. During the evolution the flow hypersurfaces stay in $\bar{\Omega}$. 
This is an immediate consequence of the Harnack inequality, cf. 4 Lemma 5.1] for details.

As a consequence of Lemma 3.1 we obtain

$$
\inf _{\mathcal{S}_{0}} u_{1} \leq u \leq \sup _{\mathcal{S}_{0}} u_{2} \quad \forall t \in I .
$$

We are now able to derive the $C^{1}$-estimates, i.e. we shall show that the hypersurfaces remain uniformly space-like, or equivalently, that the term

$$
\tilde{v}=v^{-1}=\frac{1}{\sqrt{1-|D u|^{2}}}
$$

is uniformly bounded.

Let us first derive an evolution equation for $\tilde{v}$.

3.2. Lemma (Evolution of $\tilde{v})$. Consider the flow (2.5) in the distinguished coordinate system associated with $\mathcal{S}_{0}$. Then, $\tilde{v}$ satisfies the evolution equation

$$
\begin{aligned}
\dot{\tilde{v}}-\Delta \tilde{v}= & -\|A\|^{2} \tilde{v}-f \eta_{\alpha \beta} \nu^{\alpha} \nu^{\beta}-f_{\beta} x_{i}^{\beta} \eta_{\alpha} x_{k}^{\alpha} g^{i k} \\
& -2 h^{i j} x_{i}^{\alpha} x_{j}^{\beta} \eta_{\alpha \beta}-g^{i j} \eta_{\alpha \beta \gamma} x_{i}^{\beta} x_{j}^{\gamma} \nu^{\alpha} \\
& -\bar{R}_{\alpha \beta} \nu^{\alpha} x_{k}^{\beta} \eta_{\gamma} x_{l}^{\gamma} g^{k l},
\end{aligned}
$$

where $\eta$ is the covariant vector field $\left(\eta_{\alpha}\right)=e^{\psi}(-1,0, \ldots, 0)$.

Proof. We have $\tilde{v}=\langle\eta, \nu\rangle$. Let $\left(\xi^{i}\right)$ be local coordinates for $M(t)$. Differentiating $\tilde{v}$ covariantly we deduce

$$
\begin{gathered}
\tilde{v}_{i}=\eta_{\alpha \beta} x_{i}^{\beta} \nu^{\alpha}+\eta_{\alpha} \nu_{i}^{\alpha}, \\
\tilde{v}_{i j}=\eta_{\alpha \beta \gamma} x_{i}^{\beta} x_{j}^{\gamma} \nu^{\alpha}+\eta_{\alpha \beta} x_{i j}^{\beta} \nu^{\alpha} \\
+\eta_{\alpha \beta} x_{i}^{\beta} \nu_{j}^{\alpha}+\eta_{\alpha \beta} x_{j}^{\beta} \nu_{i}^{\alpha}+\eta_{\alpha} \nu_{i j}^{\alpha}
\end{gathered}
$$

The time derivative of $\tilde{v}$ can be expressed as

$$
\begin{aligned}
\dot{\tilde{v}} & =\eta_{\alpha \beta} \dot{x}^{\beta} \nu^{\alpha}+\eta_{\alpha} \dot{\nu}^{\alpha} \\
& =\eta_{\alpha \beta} \nu^{\alpha} \nu^{\beta}(H-f)+(H-f)^{k} x_{k}^{\alpha} \eta_{\alpha} \\
& =\eta_{\alpha \beta} \nu^{\alpha} \nu^{\beta}(H-f)+H^{k} x_{k}^{\alpha} \eta_{\alpha}-f_{\beta} x_{i}^{\beta} x_{k}^{\alpha} g^{i k} \eta_{\alpha},
\end{aligned}
$$

where we have used (2.7).

Substituting (3.11) and (3.12) in (3.9), and simplifying the resulting equation with the help of the Weingarten and Codazzi equations, we arrive at the desired conclusion. 
3.3. Lemma. There is a constant $c=c(\Omega)$ such that for any positive function $0<\epsilon=\epsilon(x)$ on $\mathcal{S}_{0}$ and any hypersurface $M(t)$ of the flow we have

$$
\begin{aligned}
\|\nu\| & \leq c \tilde{v}, \\
g^{i j} & \leq c \tilde{v}^{2} \sigma^{i j},
\end{aligned}
$$

and

$$
\left|h^{i j} \eta_{\alpha \beta} x_{i}^{\alpha} x_{j}^{\beta}\right| \leq \frac{\epsilon}{2}\|A\|^{2} \tilde{v}+\frac{c}{2 \epsilon} \tilde{v}^{3}
$$

where $\left(\eta_{\alpha}\right)$ is the vector field in Lemma 3.2.

Proof. The first two estimates can be immediately verified. To prove (3.15) we choose local coordinates $\left(\xi^{i}\right)$ such that

$$
h_{i j}=\kappa_{i} \delta_{i j}, \quad g_{i j}=\delta_{i j}
$$

and deduce

$$
\begin{aligned}
\left|h^{i j} \eta_{\alpha \beta} x_{i}^{\alpha} x_{j}^{\beta}\right| & \leq \sum_{i}\left|\kappa_{i}\right|\left|\eta_{\alpha \beta} x_{i}^{\alpha} x_{i}^{\beta}\right| \\
& \leq \frac{\epsilon}{2}\|A\|^{2} \tilde{v}+\frac{1}{2 \epsilon} \tilde{v}^{-1} \sum_{i}\left|\eta_{\alpha \beta} x_{i}^{\alpha} x_{i}^{\beta}\right|^{2},
\end{aligned}
$$

and

$$
\sum_{i}\left|\eta_{\alpha \beta} x_{i}^{\alpha} x_{i}^{\beta}\right|^{2} \leq g^{i k} \eta_{\alpha \beta} x_{i}^{\alpha} x_{j}^{\beta} g^{j l} \eta_{\gamma \delta} x_{k}^{\gamma} x_{l}^{\delta} .
$$

Hence, the result in view of (3.14).

Combining the preceding lemmata we infer

3.4. Lemma. There is a constant $c=c(\Omega)$ such that for any positive function $\epsilon=\epsilon(x)$ on $\mathcal{S}_{0}$ the term $\tilde{v}$ satisfies a parabolic inequality of the form

$$
\dot{\tilde{v}}-\Delta \tilde{v} \leq-(1-\epsilon)\|A\|^{2} \tilde{v}+c[|f|+\|D f \mid\|] \tilde{v}^{2}+c\left[1+\epsilon^{-1}\right] \tilde{v}^{3} .
$$

We note that the statement $c$ depends on $\Omega$ also implies that $c$ depends on geometric quantities of the ambient space restricted to $\Omega$.

We further need the following two lemmata

3.5. Lemma. Let $M(t)=$ graph $u(t)$ be the flow hypersurfaces, then we have

$$
\dot{u}-\Delta u=e^{-\psi} v^{-1} f-e^{-\psi} g^{i j} \bar{h}_{i j}+\bar{\Gamma}_{00}^{0}\|D u\|^{2}+2 \bar{\Gamma}_{0 i}^{0} u^{i},
$$

where the time derivative is a total derivative. 
Proof. We use the relation

$$
\dot{u}=-e^{-\psi} v^{-1}(H-f)
$$

together with (1.16).

3.6. Lemma. Let $M \subset \bar{\Omega}$ be a graph over $\mathcal{S}_{0}, M=\operatorname{graph} u$, then

$$
\left|\tilde{v}_{i} u^{i}\right| \leq c \tilde{v}^{3}+\|A\| e^{\psi}\|D u\|^{2},
$$

where $c=c(\Omega)$.

Proof. First, we use that

$$
\tilde{v}^{2}=1+e^{2 \psi}\|D u\|^{2}
$$

and thus,

$$
2 \tilde{v} \tilde{v}_{i}=2 \psi_{\alpha} x_{i}^{\alpha} e^{2 \psi}\|D u\|^{2}+2 e^{2 \psi} u_{i j} u^{j},
$$

from which we infer

$$
\left|\tilde{v}_{i} u^{i}\right| \leq c \tilde{v}^{3}+\tilde{v}^{-1} e^{2 \psi}\left|u_{i j} u^{i} u^{j}\right|,
$$

which gives the result because of (1.16).

We are now ready to prove the uniform boundedness of $\tilde{v}$.

3.7. Proposition. During the evolution the term $\tilde{v}$ remains uniformly bounded

$$
\tilde{v} \leq c=c(\Omega,|f|,\|D f \mid\|) .
$$

Proof. Let $\mu, \lambda$ be positive constants, where $\mu$ is supposed to be small and $\lambda$ large, and define

$$
\varphi=e^{\mu e^{\lambda u}}
$$

where we assume without loss of generality that $1 \leq u$, otherwise replace in (3.27) $u$ by $(u+c), c$ large enough.

We shall show that

$$
w=\tilde{v} \varphi
$$

is uniformly bounded if $\mu, \lambda$ are chosen appropriately.

In view of Lemma 3.3 and Lemma 3.5 we have

$$
\dot{\varphi}-\Delta \varphi \leq c \mu \lambda e^{\lambda u}\left[\tilde{v}|f|+\tilde{v}^{2}\right] \varphi-\mu \lambda^{2} e^{\lambda u}\left[1+\mu e^{\lambda u}\right]\|D u\|^{2} \varphi,
$$


from which we further deduce taking Lemma 3.4 and Lemma 3.6 into account

$$
\begin{aligned}
\dot{w}-\Delta w \leq & -(1-\epsilon)\|A\|^{2} \tilde{v} \varphi+c[|f|+\|D f\|] \tilde{v}^{2} \varphi \\
& +c\left[1+\epsilon^{-1}\right] \tilde{v}^{3} \varphi-\mu \lambda^{2} e^{\lambda u}\left[1+\mu e^{\lambda u}\right] \tilde{v}\|D u\|^{2} \varphi \\
& +c[1+|f|] \mu \lambda e^{\lambda u} \tilde{v}^{3} \varphi+2 \mu \lambda e^{\lambda u}\|A\| e^{\psi}\|D u\|^{2} \varphi .
\end{aligned}
$$

We estimate the last term on the right-hand side by

$$
\begin{aligned}
2 \mu \lambda e^{\lambda u}\|A\| e^{\psi}\|D u\|^{2} \varphi \leq & (1-\epsilon)\|A\|^{2} \tilde{v} \varphi \\
& +\frac{1}{1-\epsilon} \mu^{2} \lambda^{2} e^{2 \lambda u} \tilde{v}^{-1} e^{2 \psi}\|D u\|^{4} \varphi,
\end{aligned}
$$

and conclude

$$
\begin{aligned}
\dot{w}-\Delta w \leq & c[|f|+\|D f\| \|] \tilde{v}^{2} \varphi+c[1+|f|] \mu \lambda e^{\lambda u} \tilde{v}^{3} \varphi \\
& +c\left[1+\epsilon^{-1}\right] \tilde{v}^{3} \varphi+\left[\frac{1}{1-\epsilon}-1\right] \mu^{2} \lambda^{2} e^{2 \lambda u}\|D u\|^{2} \tilde{v} \varphi \\
& -\mu \lambda^{2} e^{\lambda u}\|D u\|^{2} \tilde{v} \varphi
\end{aligned}
$$

where we have used that

$$
e^{2 \psi}\|D u\|^{2} \leq \tilde{v}^{2} .
$$

Setting $\epsilon=e^{-\lambda u}$, we then obtain

$$
\begin{aligned}
\dot{w}-\Delta w \leq & c[|f|+|| D f \mid \|] \tilde{v}^{2} \varphi+c e^{\lambda u} \tilde{v}^{3} \varphi \\
& +c[1+|f|] \mu \lambda e^{\lambda u} \tilde{v}^{3} \varphi \\
& +\left[\frac{\mu}{1-\epsilon}-1\right] \mu \lambda^{2} e^{\lambda u}\|D u\|^{2} \tilde{v} \varphi .
\end{aligned}
$$

Now, we choose $\mu=\frac{1}{2}$ and $\lambda_{0}$ so large that

$$
\frac{\mu}{1-e^{-\lambda u}} \leq \frac{3}{4} \quad \forall \lambda \geq \lambda_{0}
$$

and infer that the last term on the right-hand side of (3.34) is less than

$$
-\frac{1}{8} \lambda^{2} e^{\lambda u}\|D u\|^{2} \tilde{v} \varphi
$$

which in turn can be estimated from above by

$$
-c \lambda^{2} e^{\lambda u} \tilde{v}^{3} \varphi
$$

at points where $\tilde{v} \geq 2$. 
Thus, we conclude that for

$$
\lambda \geq \max \left(\lambda_{0}, 4\left[1+|f|_{\Omega}\right]\right)
$$

the parabolic maximum principle, applied to $w$, yields

$$
w \leq \operatorname{const}\left(|w(0)|_{\mathcal{S}_{0}}, \lambda_{0},|f|,|| D f \mid \|, \Omega\right) .
$$

\section{4. $C^{2}$-estimates}

Since the mean curvature operator is a quasilinear operator, the uniform $C^{1}$-estimates we have established in the last section also yield uniform $C^{2}$-estimates during the evolution, but nevertheless, we would like to give an independent proof of the $C^{2}$-estimates.

4.1. Lemma. During the evolution the principal curvatures of the evolution hypersurfaces $M(t)$ are uniformly bounded.

Proof. As already mentioned in Remark 2.6. we know that $f \leq H$, thus, it is sufficient to estimate the principal curvatures from above.

Let $\varphi$ be defined by

$$
\varphi=\sup \left\{h_{i j} \eta^{i} \eta^{j}:\|\eta\|=1\right\} .
$$

We claim that $\varphi$ is uniformly bounded.

Let $0<T<T^{*}$, and $x_{0}=x_{0}\left(t_{0}\right)$, with $0<t_{0} \leq T$, be a point in $M\left(t_{0}\right)$ such that

$$
\sup _{M_{0}} \varphi<\sup \left\{\sup _{M(t)} \varphi: 0<t \leq T\right\}=\varphi\left(x_{0}\right) .
$$

We then introduce a Riemannian normal coordinate system $\left(\xi^{i}\right)$ at $x_{0} \in M\left(t_{0}\right)$ such that at $x_{0}=x\left(t_{0}, \xi_{0}\right)$ we have

$$
g_{i j}=\delta_{i j} \quad \text { and } \quad \varphi=h_{n}^{n} .
$$

Let $\tilde{\eta}=\left(\tilde{\eta}^{i}\right)$ be the contravariant vector field defined by

$$
\tilde{\eta}=(0, \ldots, 0,1),
$$

and set

$$
\tilde{\varphi}=\frac{h_{i j} \tilde{\eta}^{i} \tilde{\eta}^{j}}{g_{i j} \tilde{\eta}^{i} \tilde{\eta}^{j}} .
$$

$\tilde{\varphi}$ is well defined in neighbourhood of $\left(t_{0}, \xi_{0}\right)$, and $\tilde{\varphi}$ assumes its maximum at $\left(t_{0}, \xi_{0}\right)$. Moreover, at $\left(t_{0}, \xi_{0}\right)$ we have

$$
\dot{\tilde{\varphi}}=\dot{h}_{n}^{n},
$$


and the spatial derivatives do also coincide; in short, at $\left(t_{0}, \xi_{0}\right) \tilde{\varphi}$ satisfies the same differential equation (2.13) as $h_{n}^{n}$. For the sake of greater clarity, let us therefore treat $h_{n}^{n}$ like a scalar and pretend that $\varphi=h_{n}^{n}$.

At $\left(t_{0}, \xi_{0}\right)$ we have $\dot{\varphi} \geq 0$, and, in view of the maximum principle, we deduce from Lemma 2.5

$$
0 \leq-\|A\|^{2} h_{n}^{n}+f\left|h_{n}^{n}\right|^{2}+c\left[|f|+\|D f \mid\|+\left\|D^{2} f\right\| \|\right]\left[1+\left|h_{n}^{n}\right|\right] .
$$

Thus, $\varphi$ is uniformly bounded.

\section{Convergence to a stationary solution}

We are now ready to give a new proof of Theorem 2.2. Let us look at the scalar version of the flow as in (3.6)

$$
\frac{\partial u}{\partial t}=-e^{-\psi} v(H-f) .
$$

This is a scalar parabolic differential equation defined on the cylinder

$$
Q_{T^{*}}=\left[0, T^{*}\right) \times \mathcal{S}_{0}
$$

with initial value $u(0)=u_{2} \in C^{4, \alpha}\left(\mathcal{S}_{0}\right)$. In view of the a priori estimates, which we have established in the preceding sections, we know that

$$
|u|_{2,0, \mathcal{S}_{0}} \leq c
$$

and

$$
H \text { is uniformly elliptic in } u
$$

independent of $t$. Thus, we can apply the known regularity results, see e.g. 11, Chapter 5.5], where even more general operators are considered, to conclude that uniform $C^{2, \alpha}$-estimates are valid, leading further to uniform $C^{4, \alpha}$-estimates due to the regularity results for linear operators.

Therefore, the maximal time interval is unbounded, i.e. $T^{*}=\infty$.

Now, integrating (5.1) with respect to $t$, and observing that the righthand side is non-positive, yields

$$
u(0, x)-u(t, x)=\int_{0}^{t} e^{-\psi} v(H-f) \geq c \int_{0}^{t}(H-f),
$$

i.e.,

$$
\int_{0}^{\infty}|H-f|<\infty \quad \forall x \in \mathcal{S}_{0}
$$

Hence, for any $x \in \mathcal{S}_{0}$ there is a sequence $t_{k} \rightarrow \infty$ such that $(H-f) \rightarrow 0$.

On the other hand, $u(\cdot, x)$ is monotone decreasing and therefore

$$
\lim _{t \rightarrow \infty} u(t, x)=\tilde{u}(x)
$$


exists and is of class $C^{4, \alpha}\left(\mathcal{S}_{0}\right)$ in view of the a priori estimates. We, finally, conclude that $\tilde{u}$ is a stationary solution of our problem, and that

$$
\lim _{t \rightarrow \infty}(H-f)=0 .
$$

To prove existence under the weaker assumptions of Theorem 2.2 we use approximation and the a priori estimate in [3, Theorem 4.1].

\section{REFERENCES}

[1] Bartnik, R.: Existence of maximal surfaces in asymptotically flat spacetimes, Commun. Math. Phys. 94, 155-175 (1984)

[2] Ecker,K., Huisken, H.: Parabolic methods for the construction of spacelike slices of prescribed mean curvature in cosmological spacetimes, Comm. Math. Phys. 135, 595-613 (1991)

[3] Gerhardt, C.: H-surfaces in Lorentzian manifolds, Commun. Math. Phys. 89, 523-553 (1983)

[4] _ Closed Weingarten hypersurfaces in Riemannian manifolds, J. Differential Geom. 43, 512-641 (1996), download pdf file

[5] - Hypersurfaces of prescribed Weingarten curvature, Math. Z. 224, 167-194 (1997) download pdf file

[6] _ Hypersurfaces of prescribed curvature in Lorentzian manifolds, Indiana Univ. Math. J. 49 (2000), 1125-1153, arXiv:math.DG/0409457

[7] _ On the foliation of space-time by constant mean curvature hypersurfaces, 7 pages, math.DG/0304423

[8] Geroch, R.P.: The domain of dependence, J. Math. Phys. 11, 437-449 (1970)

[9] Geroch, R. P., Horowitz, G.T.: Global structure of space-time. In: S. W. Hawking \& W. Israel: General Relativity. An Einstein centenary survey, pp. 212-293, Cambridge: Cambridge University Press 1979

[10] Hawking, S.W., Ellis, G.F.R.: The large scale structure of space-time, Cambridge: Cambridge University Press 1973.

[11] Krylov, N.V.: Nonlinear elliptic and parabolic equations of second order, Dordrecht: Reidel 1987.

Ruprecht-Karls-Universität, Institut für Angewandte Mathematik, Im Neuenheimer Feld 294, 69120 Heidelberg, Germany

E-mail address: gerhardt@math.uni-heidelberg.de 